\title{
Optimum Location of DG Units Considering Operation Conditions
}

\author{
Soheil Dolatiary \\ Islamic Azad University \\ Central of Tehran,
}

Tehran, Iran.

\author{
Javad Rahmani \\ Digital electronics Engineering \\ Islamic Azad University \\ Science and Research Branch
}

\author{
Zahra Khalilzad \\ Digital electronics department, \\ Shahed university of Tehran \\ Tehran, Iran.
}

Tehran, Iran.

\begin{abstract}
The optimal sizing and placement of Distributed Generation units (DG) are becoming very attractive to researchers these days. In this paper a two stage approach has been used for allocation and sizing of DGs in distribution system with time varying load model. The strategic placement of DGs can help in reducing energy losses and improving voltage profile. The proposed work discusses time varying loads that can be useful for selecting the location and optimizing DG operation. The method has the potential to be used for integrating the available DGs by identifying the best locations in a power system. The proposed method has been demonstrated on 9-bus test system.
\end{abstract}

Keywords: Distribution Generation (DG), Optimal placement, Time-varying load, Load profile, Energy losses

\section{INTRODUCTION}

In the restructured power systems, in order to considering losses, distributed generation units have been spread out in the power distribution systems. In the literature review, DG is a small scale power generation that is usually connected to a distribution system. DGs mainly consist of renewable energy resources such as photovoltaics (PV), wind turbines, and fuel cells. Among them PVs and fuel cells are DC resources and they are connecting to the power grid by DC/DC and DC/AC converters. DC/DC converters are mainly used to provide a controlled output voltage under different load variation [1]. The term DG also implies the use of generation units to lower the cost of services. A potential advantage of DG over conventional generations is that the energy production takes place near the consumer, which can minimize the power losses in the distribution lines.

Naresh Acharya et al suggested a heuristic method in [2] to select appropriate location and to calculate DG size for minimum real power losses. Though the method is effective in selecting location, it requires more computational efforts. The optimal value of DG for minimum system losses is calculated at each bus. Placing the calculated DG size for the buses one by one, corresponding system losses are calculated and compared to decide the appropriate location. Moreover, the heuristic search requires exhaustive search for all possible locations which may not be applicable to more than one DG. This method is used to calculate DG size based on approximate loss formula may lead to an inappropriate solution [3].

Go swami et al, [4] have analyzed load voltage sensitivity considering load models of voltage dependent load, whereas this method has been done by genetic algorithm. Singh et al [5] unlike other studies which dealt with the constant load models have studied on the effect of different load and sensitive to voltage and frequency and then they found the best location for DG units.
Authors of [6] have used and analytical method for optimal DG allocation, this method is based on power flow for the radial network and calculate loss sensitivity factor and priority list, which causes reduction on the search space. In [7] are proposed a heuristic method for optimal sizing and placement of DG in order to reduce economic costs, like energy cost, investment, operational cost, loss cost and technical aspect such as energy loss and voltage level.

In [8] optimal DG location is obtained considering economic and operational limits of DG and distribution system. Optimal DG placement is accomplished in [9] to maximize DG application benefit and minimize the costs for both utility and customers. A loss minimization approach is used in [10] too find optimal DG location.

There are so many DG placement methods in hand though each of these methods only focuses on some parameters. The optimal DG placement defined in [11] takes reliability, loss reduction, and load prediction into account while it fails to account for other parameters such as productivity, cost effectiveness, and type of DG. The optimal DG placement defined in [12] takes productivity, cost, effectiveness, loss reduction, and reliability and DG type into account and fails to consider other parameters.

In [13], a Newton-Raphson algorithm based load flow program is used to solve the load flow problem. The methodology for optimal placement of only one DG type 1 is proposed. Moreover, the heuristic search requires exhaustive search for all possible locations which may not be applicable to more than one DG. Therefore, in this paper, PSO method is proposed to determine the optimal location and sizes of multiDGs to minimize total real power loss of distribution systems.

Whether DG is properly planned and operated it may provide benefits to distribution networks (e.g., reduction of power losses and/or deferment of investments for network enforcing, etc.), otherwise it can cause degradation of power quality, reliability, and control of the power system [14]. Also, placement of different DG units in the power system should 
be done considering interactions of the different units under primary and secondary frequency regulation carefully to prevent power system instability [15]. Thus, DG offers an alternative that planners should explore in their search for the best solution to electric supply problems and requires new planning paradigms and procedures able to face a more complex and uncertain scenario [16], [17], [18], [19].

Ault et al. in [20] have pointed out the dichotomy between the advanced status of academic researches on planning and the unwillingness of companies to resort to such algorithms. Indeed, the planners need tools to deal with uncertainties, risks, and multiple criteria. The final choice will be subjectively operated in the set of good solutions. To consider uncertainties of parameters and system inputs, [21] uses reachability analysis which is a mathematical analysis based on uncertain matrices. Reachability analysis can also be used to study DG integration and planning uncertainties.

An optimization technique should be employed for the design of engineering systems, allowing for the best allocation of limited financial resources. In electric power systems, most of the electrical energy losses occur in the distribution systems. It is a tool that can be used both for the design of a new distribution system and for the resizing of an existing system [22]. In [23] application of robust MPC provides an optimal solution to handle the system uncertainties. In [24] MonteCarlo method is used to take uncertainties into account for renewable generation.

Distributed generation is not limited to conventional generation of electricity. A controlled reduction in demand can play the same role as distributed generation. For instance, [25] studies the possibility of using aggregation of small $\{\mathrm{ON} / \mathrm{OFF}\}$ loads as a compensation for renewables variations. It was shown that only in Texas, $1.5 \mathrm{GW}$ of flexible demand can act as distributed generation when controlled over the WiFi network.

Distributed Generation sometimes provides the most economical solution to load variations. Under voltages or overloads that are created by load growth may only exist on the circuit for a small number of hours per day or/ month or/ year. There may be many locations where DGs can be located and provide the necessary control [26]. Also, the authors address this issue for small size distributed generators in [27]. In [28] UPFC acts like a DG, and leads to power swing reduction and enhancing the system stability by reactive power injection.

However, the current research lacks the complete solution to the determination of DG allocation and operation, together with considering load voltage profile and time varying loads. For example too much emphasis on power loss cost and construction expenses but ignorance of the time varying loads and energy loss factors.

Moreover, the absence of the consideration about operation of DGs in the network structure may lead to the confusion of the benefit of placing DGs and dispatching policy.
The optimal placement and dispatching investigated in this paper are divided into two major parts, namely optimal allocation and optimal sizing of DGs. In firs part of section 2, placement of DGs is done based on the summation of energy losses during 24 hour and for time varying loads. In second part of section 2 sizing of DGs is determined in each time interval. In section 3 simulation network is explained. Simulation results on the test system are illustrated in section 4. Then the conclusion is given in section 5 .

\section{PROPOSED METHOD}

\subsection{Optimal Allocation of DGs}

Optimal DG placement achieved to gain the optimal DG location to minimize or maximize a particular objective function. The optimal allocation model of DG is used to solve the problem of sizing and siting of various DGs $[29,30]$.

Here, an energy based approach is explained for placement of a conventional DG in a distribution system. In this work conventional DGs are supposed to be diesel generators and fossil fuels producers. Wind turbines and solar panels have some limitations that cannot be used everywhere in distribution network and should be place in some particular locations. By considering these facts, only conventional DGs will be placed.

Loads that are studied here, are time varying that have different values at each time of day and night. Thus, the goal is minimizing the energy loss of distribution network. Load model are usually considered to be constant in a time period, while in reality, loads are function of the ambient temperature and human behavior $[31,32]$

So, considering energy losses instead of power losses seems better and therefore, minimizing the energy losses is the final goal. Using medium-voltage and high-voltage dc collection system would further improve the efficiency [33]. In [34] and [35], a new method for application in communication circuit system is proposed that it causes increasing the efficiency, PAE, output power and gain.

In order to minimizing the energy losses, load modeling is inevitable. As the load varies along time in 24 hour of a day, the time period should be divided into some appropriate intervals in order to approximate the load to be constant in each interval based on load profile. For instance, load profile in 24 hours is considered as follows:

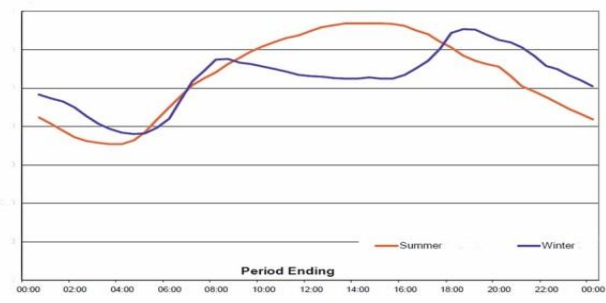

Figure 1: Load profile in 24 hours

Considering magnitude and variation of load during 24 hours of a day, sometime-intervals seem appropriate for the proposed method. Depending on the accuracy needed in a 
power network, the number of time intervals are computed. In each interval, load is being estimated by the average of load during at that interval with trapezoid method.

It should be noted that the duration of time intervals should not be the same. For example in the first times of a day that the demands are low and the variation is low enough to be neglected, the duration of that interval could be large enough to estimate the load at that interval. Although, in peak hours the variation of demands are high and the time interval should be considered small.

Here, in each interval in order to reduce energy losses of total network, the generation energy of each bus is computed. The proposed energy loss factor due to line currents can be defined as:

$$
\text { Energy loss }=\sum_{j=1}^{T} \sum_{k=1}^{N} R_{k} I_{k j}^{2} t_{j}
$$

Where:

$\mathrm{R}_{\mathrm{k}}$ are the $\mathrm{k}^{\text {th }}$ line total resistance.

$\mathrm{N}$ is the number of lines in the network.

$\mathrm{T}$ is the number of intervals for load modeling.

$\mathrm{I}_{\mathrm{kj}}$ is the current for $\mathrm{k}^{\text {th }}$ line in $\mathrm{j}^{\text {th }}$ interval.

$t_{j}$ is the duration of $k^{\text {th }}$ interval.

With this in mind, the optimization objective and the constraints are as follows:

$$
\begin{gathered}
\min \left\{\text { energy } \operatorname{loss}_{j}=\sum_{k=1}^{N} R_{k} I_{k j}^{2} t_{j}\right\} \\
\text { s.t: } V_{\text {bus }}^{\min } \leq V_{\text {bus }} \leq V_{\text {bus }}^{\max }
\end{gathered}
$$

Where, $V_{b u s}^{\min }=0.95$ p.u. and $V_{b u s}^{\max }=1.05$ p.u.

In this section, the goal is siting the DGs in special buses in order to minimizing the energy loss. For this purpose, it is assumed that in each bus of the network, there is possibility to generate electrical energy and so, DGs could be place anywhere in the power network.

Power generated in each interval by each DG could be varying from $0 \mathrm{MW}$ to the total load at that interval. The step of variation is considered $2 \mathrm{MW}$. In other words, DGs are available at sizes of $2 \mathrm{MW}$ and in each bus, there is possibility to place any number of DGs.

The result of optimizing this problem is the power generated by each DG at each interval. The output power of each DG is given by:

$$
P_{i j}=K_{i j} * 2
$$

Where, $\mathrm{K}_{\mathrm{ij}}$ is the number of $2 \mathrm{MW}$ steps in $\mathrm{i}^{\text {th }}$ bus and $\mathrm{j}^{\text {th }}$ time interval. The total energy produced by each bus during the 24 hours is as follows:

$$
E_{i}=\sum_{j=1}^{T} E_{i j}=\sum_{j=1}^{T} P_{i j} * t_{j}
$$

As there is limitation in producing energy at all buses, selecting the buses that generate more energy is important. Therefore, by selecting the buses with bigger $E_{i}$ and based on the number of buses needed for generating energy, placement of DGs is finished. For example if just 4 buses have this ability to be placed by DGs, 4 buses with higher total energy producing will be selected.

\subsection{Optimal Sizing of DGs}

In this section, after optimal DG placement and selecting the generator buses, the power generated by each DG in the selected buses for each time interval should be determined. In other words, Operation of DGs contributes in minimizing the total energy losses of the network. Thus, scheduling the energy produced by each DG should be done.

For this purpose, the minimizing objective is as mentioned before. The difference is just the number of buses producing energy. In the previous section, the placement of DGs is done in all the buses of the network and based on the total energy produced in each bus, buses with higher $\mathrm{E}_{\mathrm{i}}$ was selected. In this section, placement of DGs is just done in the selected buses of the previous section.

In optimizing the size of the DGs in order to improve computational accuracy, steps of variation in the power generated is considered 0.5 MW. In other words, DGs are available at sizes of $0.5 \mathrm{MW}$ and in each bus, there is possibility to place any number of DGs. It should be considered that increasing the steps of variation is possible, due to reduction in the number of buses generating energy and so the computational time will not increase so much.

With this consideration, the objective function and optimization problem is as mentioned before. The difference is just the output. The output of this optimization is generated power matrix in each bus.

The matrix is as follows:

$$
P=\left[P_{i j}\right]
$$

Where $P_{i j}$ is the generated power of $i^{\text {th }}$ bus producer energy and $j^{t h}$ time interval and is computed as follows:

$$
P_{i j}=K_{i j} \times 0.5 \mathrm{MW}
$$

In this formula, $\mathrm{K}_{\mathrm{ij}}$ is the number of $0.5 \mathrm{MW}$ steps.

According to this goal optimization, the loss reduces when DGs are placed. Also, the voltage limits that mentioned before, shows that the appropriate voltage of a certain bus depends on the minimum and maximum voltages of the bus which considered 0.95 and 1.05 p.u, respectively. 


\section{SIMULATION NETWORK}

In the proposed work, in order to implement the suggested method and compare the results with the network without DGs, a 9-bus distribution ring network has been selected as a sample. It should be noted that the specified algorithm can be used for all distribution networks with any number of buses and there is no limitation in implementing this algorithm. The single line diagram of the sample network is illustrated in figure 2 .

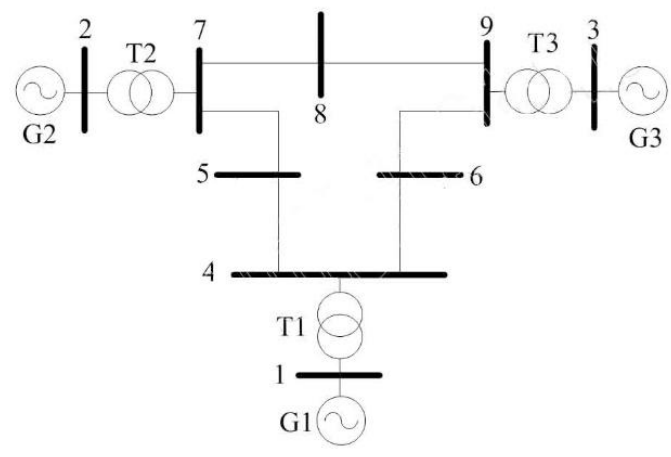

Figure 2: Single line diagram of the sample network

According to figure 2, the nine-bus system contains two sections. Section 1 is buses 1,2 , and 3 with voltage of $63 \mathrm{kV}$. These buses get connected to the other section by transformers with the ratio of 63/20. The other section is buses 4 to 9 with voltage of $20 \mathrm{kV}$. Placement of DGs is done in section 2, in the ring network with buses of $20 \mathrm{kV}$.

Table 1 shows the data of the lines of the network.

Table 1: Data of the lines of the network

\begin{tabular}{|c|c|c|}
\hline Line & $\mathrm{R}(\mathrm{pu})$ & $\mathrm{X}(\mathrm{pu})$ \\
\hline 1 & 0.01 & 0.085 \\
\hline 2 & 0.032 & 0.161 \\
\hline 3 & 0.017 & 0.092 \\
\hline 4 & 0.039 & 0.17 \\
\hline 5 & 0.085 & 0.072 \\
\hline 6 & 0.0119 & 0.1008 \\
\hline
\end{tabular}

For load modeling, it is considered that the load variation curve is as plotted in figure 1 . So, 7 time interval could be appropriate for this purpose. These time intervals are as follows:

$$
\begin{gathered}
t_{1}: t=0-5 \\
t_{2}: t=5-7 \\
t_{3}: t=7-12 \\
t_{4}: t=12-16 \\
t_{5}: t=16-20 \\
t_{6}: t=20-22 \\
t_{7}: t=22-24
\end{gathered}
$$

Figure 3 shows the load profile estimation for load number 1 in 24 hours of a day.

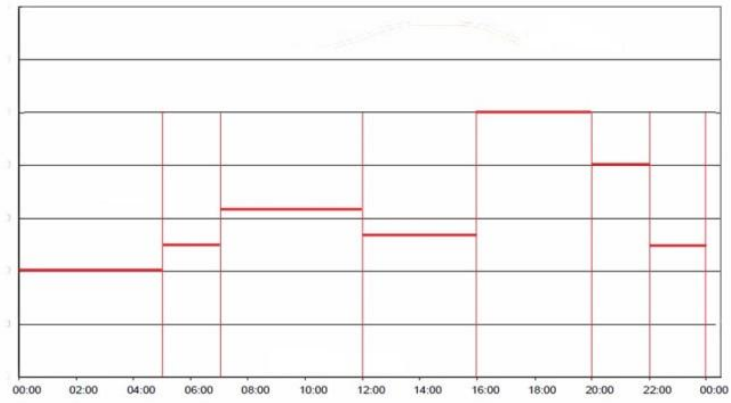

Figure 3: Load profile

In table 2, data of the loads of the system is shown.

Table 2: Data of loads of the network

\begin{tabular}{|c|c|c|c|c|c|c|}
\hline $\begin{array}{c}\text { Time } \\
\text { interva } \\
1\end{array}$ & $\begin{array}{c}\text { Load } \\
1\end{array}$ & $\begin{array}{c}\text { Load } \\
2\end{array}$ & $\begin{array}{c}\text { Load } \\
3\end{array}$ & $\begin{array}{c}\text { Load } \\
4\end{array}$ & $\begin{array}{c}\text { Load } \\
5\end{array}$ & $\begin{array}{c}\text { Load } \\
6\end{array}$ \\
\hline $0-5$ & 2 & 2 & 1 & 0.5 & 1.2 & 1 \\
\hline $5-7$ & 2.5 & 2 & 1.2 & 1 & 1.5 & 1.1 \\
\hline $7-12$ & 3.2 & 2.7 & 1.8 & 1.5 & 2 & 1.4 \\
\hline $12-16$ & 2.6 & 2.1 & 1.2 & 0.9 & 1.4 & 1.2 \\
\hline $16-20$ & 5 & 4 & 3 & 3 & 3 & 2 \\
\hline $20-22$ & 4 & 3.5 & 2.7 & 2.8 & 3 & 1.8 \\
\hline $22-24$ & 2.5 & 2.1 & 1.3 & 1.1 & 1.4 & 1.5 \\
\hline
\end{tabular}

\section{SIMULATION RESULTS}

This study aims to optimize the placement and operation of DGs by taking energy losses into account. It should be noted that siting the DGs is done in $20 \mathrm{kV}$ buses.

The peak demand in this network is 20 MW. Thus, the maximum total power generated by these 3-DG, are considered to be nearly $20 \mathrm{MW}$ in peak hours. In other time intervals, also the total energy produced by DGs is near the total load at that interval.

In the first step, the goal is the placement of DGs in the network. By implementing the proposed method on the sample network, these results are achieved.

$$
E=\left[\begin{array}{lllll}
52 & 4036 & 38 & 60 & 88
\end{array}\right]
$$

In this case, it is assumed that only 3 buses can produce energy. By this consideration, buses 1, 5 and 6 which have the biggest $E_{i}$ are selected.

In the second step, the goal is optimal operation of DGs in the selected buses. Here, the algorithm of sizing the DGs is implemented on the buses 1,5 and 6 .

The following matrix is achieved for power generated at each time interval. 


\begin{tabular}{|c|c|c|c|}
\hline Time interval & E1 & E2 & E3 \\
\hline $0-5$ & 1.5 & 2 & 2 \\
\hline $5-7$ & 1.5 & 2 & 2 \\
\hline $7-12$ & 2 & 2.5 & 3 \\
\hline $12-16$ & 1.5 & 2 & 2.5 \\
\hline $16-20$ & 2.5 & 4 & 4 \\
\hline $20-22$ & 2.5 & 3.5 & 3 \\
\hline $22-24$ & 1.5 & 2 & 2 \\
\hline
\end{tabular}

The above matrix shows that some DGs produce more energy than the other one in some intervals and in the other intervals it is vice versa. For example, DG 3 in times 7 to 16 produce more energy than DG 2, but in times 20 to 22 produce less energy than DG 2 .

By considering the results of the above matrix, it is shown that placement of the DGs should not be based on the peak demand which is done in many of the research.

This method schedules the DGs based on the minimization of energy loss and gives a generating profile to each DG.

Table 3 compares the energy losses of the proposed method with the case that sizes of DGs are equal. Table 4 compares the results with other placement of DGs. The results of the tables show that the proposed method has the optimal solution.

Table 3: Comparison of the proposed method with equally dispatch

\begin{tabular}{|c|c|}
\hline methods & Energy loss(MWh) \\
\hline Proposed method & 14.636 \\
\hline
\end{tabular}

\section{REFERENCES}

[1] P. M. Shabestari, G. B. Gharehpetian, G. H. Riahy, and S. Mortazavian, "Voltage controllers for DC-DC boost converters in discontinuous current mode," International Energy and Sustainability Conference, Framingdale, NY, 2015, pp. 1-7.

[2] Naresh Acharya, Pukar Mahat, N. Mithulanathan, "An analytical approach for DG allocation in primary distribution network", Electric Power and Energy System, vol. 28.

[3] M. Padma Lalitha, V.C. Veera Reddy, V. Usha, "Optimal DG placement for minimum real power loss in radial distribution systems using PSO", Journal of Theoretical and Applied Information Technology, pp. 107-116, 2010

[4] R.K. Singh, et al., "Optimal Allocation of Distributed Generation in Distribution Network with Voltage and Frequency Dependent Loads," ICIIS, 2008, pp. 1-5.

[5] R.K. Singh and S.K. Goswami, "Optimal Siting and Sizing of Distributed Generations in Radial and Networked Systems

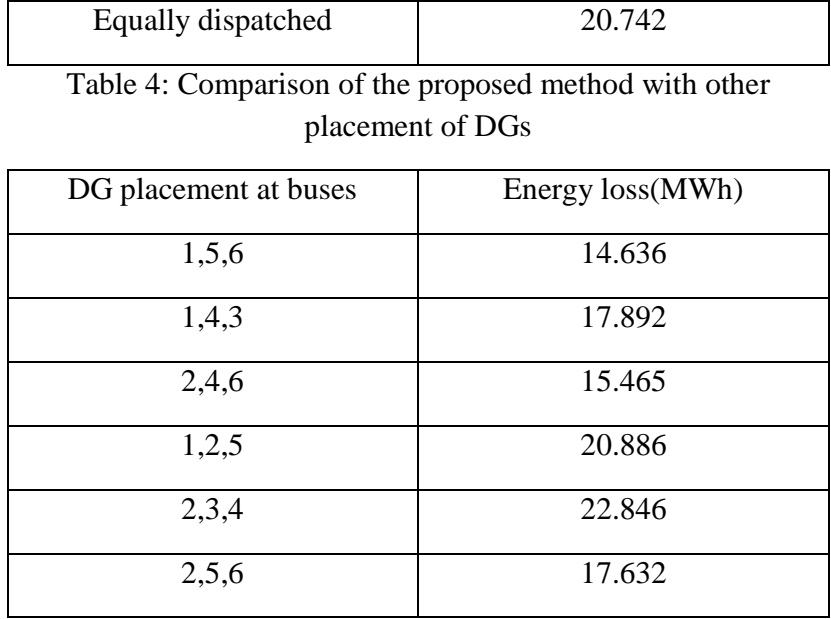

By considering the results that shown in table 3 and 4, it is obvious that placement of DGs should not be based on the peak load, which is done in many of the research.

\section{CONCLUSION}

This paper has discussed a two stage methodology of finding the optimal location and sizes of DGs for maximum energy loss reduction of distribution systems. First stage is DG placement method which is proposed to find optimal DG location. Second stage is optimal DG operation based on load varying. Voltage constraints are included in the algorithm.

This methodology is tested on 9 bus system. By installing DGs at all the potential locations and optimizing the size at each time interval, the total energy loss of the system has been reduced and the voltage profile of the system is also considered.

Inclusion of the non-linear loads and power quality constraints is the future scope of this work.

Considering Different Voltage Dependent Static Load Models," IEEE International Conference on Power and Energy, 2008, pp. 1535-1540.

[6] P. Alemi and G.B. Gharehpetian, "DG Allocation Using an Analytical Method to Minimize Losses and to Improve Voltage Security," International conference on Power and Energy, Malaysia, IEEE, 2008, pp. 1575-1580.

[7] C. Tautiva, "Optimal Placement of Distributed Generation on Distribution Networks," Universities power engineering conference, 2009, pp. 1-5.

[8] Rosehart W, Nowicki E, “ Optimal Placement of Distributed Generation", Proceeding of 14th Power Systems ComputationConference section 11, Sevilllaa 2002 ,pp.1-5

[9] Silvestri a, Berrizi A, Buonanno S, "Distributed Generation Planning Using GA”, IEEE PowerTech, 1999.

[10] Celli G, Pilo F., "Optimal Distributed Generation Allocation in MV Distribution Networks", IEEE PES Conference on Power Industry Computer Applications, 2001. 
[11] P. P. Barker and R. W. de Mello, "Determining the Impact of Distributed Generation on Power Systems. I. Ra-dial Distribution Systems," IEEE Power Engineering So-ciety Summer Meeting, Seattle, 16-20 July 2000, pp. 1645-1656.

[12] N. Hadisaid, J.-F. Canard and F. Dumas, "Dispersed Gen-eration Impact on Distribution Networks," IEEE Computer Applications in Power, Vol. 12, No. 2, 1999.

[13] Kennedy J and Eberhart R, "Particle Swarm Optimizer," IEEE International Conference on Neural Networks (Perth, Australia), IEEE Service Center Piscataway, NJ, IV,1995.

[14] N. Jenkins, R. Allan, P. Crossley, D. Kirschen, and G. Strbac, Embedded Generation. London, U.K.: IEE, 2000.

[15] M. Amini and M. Almassalkhi, "Investigating delaysin frequency-dependent load control," in InnovativeSmart Grid Technologies-Asia (ISGT-Asia), 2016 IEEE. IEEE, 2016, pp. 448-453.

[16] P. P. Barker and R.W. de Mello, "Determining the impact of distributed generation on power systems: Part 1Radial distribution systems," in Proc. IEEE Power Eng. Soc. Summer Meeting, vol. 3, Seattle, WA, Jul. 16-20, 2000.

[17] R. C. Dugan and S. K. Price, "Issues for distributed generation in the US," in Proc. IEEE Power Eng. Soc. Winter Meeting, vol. 1, New York, Jan. 2002, pp. 121-126.

[18] R. C. Dugan, T. E. McDermott, and G. J. Ball, "Planning for distributed generation,” IEEE Ind. Appl. Mag, 2001.

[19] G. W. Ault and J. R. McDonald,"Planning for distributed generation within distribution networks in restructured electricity markets,'IEEE Power Eng. Rev., 2000.

[20] M. H. Imani, et al., "Simultaneous presence of wind farm and V2G in security constrained unit commitment problem considering uncertainty of wind generation," in Texas Power and Energy Conference (TPEC), 2018, pp. 1-6.

[21] P. M. Shabestari, S. Ziaeinejad and A. Mehrizi-Sani, "Reachability analysis for a grid-connected voltage-sourced converter (VSC)," IEEE Applied Power Electronics Conference and Exposition (APEC), San Antonio, TX, March, 2018, pp. 2349-2354.

[22] Eduardo G. Carrano, et al., "Electric Distribution Network Multi objective Design using a Problem Specific Genetic Algorithm" IEEE Transactions on Power Delivery, Vol. 21, No. 2, April 2006.

[23] M. Ghanaatian; S. Lotfifard, "Control of Flywheel Energy Storage Systems in Presence of Uncertainties" IEEE Transactions on Sustainable Energy, 2018.

[24] S. Jafarishiadeh, M. Farasat "Modeling and Sizing of an Undersea Energy Storage System", IEEE Transactions on Industry Applications, vol. 54, no. 3, pp. 2727-2739, 2018.
[25] M. S. Modarresi, L. Xie, and C. Singh "Reserves from Controllable Swimming Pool Pumps: Reliability Assessment and Operational Planning," in Proc. 51st Hawaii International Conference on System Sciences (HICSS), January 2018.

[26] N. Ghanbari, H. Mokhtari, S. Bhattacharya, "Optimizing Operation Indices Considering Different Types of Distributed Generation in Microgrid Applications", Energies 2018, 11, 894.

[27] N. Ghanbari, H. Golzari, H. Mokhtari and M. Poshtan, "Optimum location for operation of small size distributed generators," IEEE International Conference on Renewable Energy Research and Applications, CA, 2017, pp. 300-303.

[28] H. Gharibpour, H. Monsef, M. Ghanaatian, "The comparison of two control methods of power swing reduction in power system with UPFC compensator" 20th Iranian Conference on Electrical Engineering (ICEE) 2012.

[29]K. Yousefpour,et al., "Dynamic Approach for Distribution System Planning Using Particle Swarm Optimization." International Journal of Control Science and Engineering.

[30] H. Pourgharibshahi, et al., "Verification of computational optimum tilt angles of a photovoltaic module using an experimental photovoltaic system," Environmental Progress \& Sustainable Energy, vol. 34, no. 4, pp. 1156-1165, 2015.

[31] M. Amini and M. Almassalkhi, "Trading off robustness and performance in receding horizon control with uncertain energy resources," in 2018 Power Systems Computation Conference (PSCC), 2018, pp. 1-7.

[32] F. Rassaei, W. Soh, K. Chua, and M.S. Modarresi, "Environmentally-friendly demand response for residential plug-in electric vehicles", in Proc. Power and Energy Conference (TPEC), College Station, TX, 2017.

[33] S. Jafarishiadeh, et al., "Grid-connected operation of direct-drive wave energy converter by using HVDC line and undersea storage system" in Proc. Energy Conversion Congress and Exposition (ECCE), Cincinnati, OH , 2017.

[34] F. Rahmani, F. Razaghian, and A. Kashaninia, "High Power Two-Stage Class-AB/J Power Amplifier with High Gain and Efficiency," Journal of Academic and Applied Studies (JAAS), vol. 4, pp. 56-68, 2014.

[35] F. Rahmani, F. Razaghian, and A. Kashaninia, "Novel Approach to Design of a Class-EJ Power Amplifier Using High Power Technology," World Academy of Science, Engineering and Technology, International Journal of Electrical, Computer, Energetic, Electronic and Communication Engineering, vol. 9, pp. 541-546, 2015. 